\title{
Characterization and comparisons of microbiota in different intestinal segments between adult Chinese Shanxi Black Pigs and Large White Pigs
}

\author{
Pengfei Gao ${ }^{1}$. Yulong Guo ${ }^{1}$. Ningfang Zhang ${ }^{1} \cdot$ Wanfeng Zhang $^{1} \cdot$ Hejie Wang ${ }^{1} \cdot$ Xiaohong Guo ${ }^{1} \cdot$ Zhibian Duan $^{1}$.

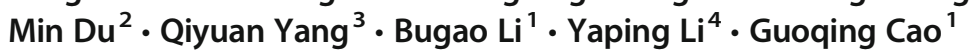

Received: 1 March 2018 / Accepted: 28 December 2018 / Published online: 14 February 2019

(C) The Author(s) 2019

\begin{abstract}
The carcass weight of Chinese Shanxi Black Pigs is relatively lower in comparison with that of the counterparts, i.e., Large White Pigs, although the former are resistant to harsh conditions. Since gut microflora has been recognized to play a key role in pork production, it is of interest to explore the microbial communities in different intestinal segments of pigs and its potential relatedness with host features. In this study, 16S rRNA gene amplicon sequencing, accompanied by the inter- and intra-group comparisons, was implemented to investigate the structure composition and potential functions of microbial communities of four distinct intestinal segments [duodenum (D), jejunum (J), ileum (I), and cecum (C)] between adult Chinese Shanxi Black Pigs and Large White Pigs. Comparative survey revealed that the dominant phylum in both breeds was Firmicutes, followed by Proteobacteria, and Bacteroidetes. At the genus level, Lactobacillus was predominant in all samples, and Prevotella was specifically prevalent in the cecum. Further inspection showed the differences of dominant species in the same segments between these two groups. Notably, unique taxa in C and D segments were more than that in I and J segments. Additionally, each segment was characterized by specifically enriched genera, and distinctive pathways were predicted in certain intestinal segments. In short, the findings presented a coherent picture of structure composition and predicted functionalities of gut microbiota in diverse intestinal segments of adult Chinese Shanxi Black Pigs and Large White Pigs, and extend the understanding of potential link between intestinal microbiota and their hosts.
\end{abstract}

Keywords Pig breeds $\cdot$ Intestinal segments $\cdot$ Gut microbiota $\cdot 16 \mathrm{~S}$ rRNA gene amplicon sequencing $\cdot$ Structure composition

Pengfei Gao and Yulong Guo contributed equally to this work.

Electronic supplementary material The online version of this article (https://doi.org/10.1007/s13213-018-1430-3) contains supplementary material, which is available to authorized users.

Guoqing Cao

997244581@qq.com

1 Department of Animal Sciences and Veterinary Medicine, Shanxi Agricultural University, Taigu 030801, China

2 Department of Animal Sciences, Washington State University, Pullman, WA 99164, USA

3 Molecular Cell and Cancer Biology Department, University of Massachusetts Medical School, Worcester, MA 01655, USA

4 Guangzhou Genedenovo Biotechnology Co., Ltd, Guangzhou 510660, China

\section{Introduction}

Currently, considerable evidences have proven that intestinal microbiota was actively involved in the digestion and absorption of nutrients in their hosts (Ley et al. 2008; Sommer and Backhed 2013; Hillman et al. 2017). Furthermore, the intestinal environments were thought to be associated with multiple diseases, such as diabetes, obesity, inflammatory bowel disease, and cancer (D'Argenio and Salvatore 2015; Barko et al. 2017; Kundu et al. 2017; Tian et al. 2017). The production capacities of lean meat and fat were considered as crucial factors in the pork industry, and have been intensively studied for several decades using various methods, such as dietary modification, genome investigation, and intervention with prebiotics (George et al. 2007; He et al. 2017; Zou et al. 2017; Lee et al. 2018). Taken together, studies on intestinal microbiota may provide some valuable references for promoting the healthy growth of pigs. 
The Chinese Shanxi Black Pig is a local pig breed, which is a hybrid of the American Duroc Pig, British Berkshire Pig, Chinese Neijiang Pig, and Chinese Mashen Pig. Of these pig breeds, the former three pigs were male parents, and the latter pig was female parent. It is mainly distributed in Shanxi Province in China, with rugged terrain at an altitude of 1$1.5 \mathrm{~km}$. The average annual temperature of the region is $4.5-8.5^{\circ} \mathrm{C}$, and annual precipitation is $400-550 \mathrm{~mm}$. The Chinese Shanxi Black Pig is regarded to be highly adaptable to harsh conditions, which is well reflected in its rapid growth. Nevertheless, it is not as well accepted as the Large White Pig, due to its relatively lower capacity and quality of carcass meat. The Large White Pig, a Western breed, was characterized by rapid growth, high rate of feed conversion, but poor resistance to disease. The reasons why the black pigs are highly adapted to the harsh settings and the white pigs harbor the higher lean meat and fat capacities remain unclear, but there is no doubt that the porcine genomes are responsible for their differentiating features. In spite of this, we could not exclude the possibility that minor yet special compositional differences in intestinal microbiota might exert beneficial effects.

Generally, 16S rRNA is a highly conserved prokaryotic ribosomal component that binds to the Shine-Dalgarno sequence (Woo et al. 2008; Ellegaard and Engel 2016; Fang et al. 2017; Inatomi et al. 2017; Kim et al. 2018), and is widely used for phylogeny and taxonomic assignments. In order to analyze and compare the microbial compositions of different intestinal segments both in Chinese Shanxi Black Pigs and Large White Pigs, 16S rRNA gene amplicon sequencing was performed using three adult Chinese Shanxi Black Pigs and three Large White Pigs at the rapid growth stage (usually 150th day). With the expectation of revealing intriguing differences, characterization and comparative survey revealed the structure composition of microbial communities in distinct intestinal compartments, including duodenum (D), jejunum $(\mathrm{J})$, ileum (I), and cecum (C), and enhance our understanding of the potential roles of intestinal microbial communities in resistance ability and meat and fat production.

\section{Materials and methods}

\section{Animals and sampling}

The experimental protocols, Good Experimental Practices, were adopted by the College of Animal Science and Veterinary Medicine, Shanxi Agricultural University, and were used for this study. Three Chinese Shanxi Black Pigs and three Large White Pigs were administrated and fed at the Datong Pig Breeding Farm (Shanxi, China) under the same conditions. At different growth stages, including lactation stage, nursing stage, and growing-finishing stage, these two breeds at various weight levels were provided with standard dietaries for nutrient requirements, which were in accordance with the feeding standard of swine (NY/T 65 2004) issued by The Ministry of Agriculture of the People's Republic of China. Although the number of individuals was relatively lower compared to those large-scale samples, it might be reasonable, to some extent, to support the statistical analysis in light of the acceptable number. The animals were fasted for $24 \mathrm{~h}$ with free access to fresh water, and were then sacrificed at the rapid growth stage (150th day) at the Datong designated pig slaughter management office according to the standard procedures. The pigs were dissected for sampling of the contents of different intestinal segments, including duodenum (D), jejunum (J), ileum (I), and cecum (C). Samples for Shanxi Black Pig and Large White Pig were labeled as B and $\mathrm{W}$, respectively. The samples were indexed with an initial letter representing the segment and an Arabic numeral for individual recognition. For instance, B I1 indicates ileal sample of pig 1 in the black pig group. The samples were immediately frozen within liquid nitrogen, and then stored at $20{ }^{\circ} \mathrm{C}$. All animals used for this study were in consistence with the Guidelines for the Care and Use of Laboratory Animals established by Laboratory Animal Center of Shanxi Agriculture University, and approved by the Animal Ethics Committee of Shanxi Agriculture University.

\section{DNA extraction, PCR amplification, and 16s rRNA gene sequencing}

To examine the microbiota in each sample, 16S rRNA genes were amplified and sequenced. For this, gut contents were initially treated with phenol-chloroform to extract genomic DNA. Then, genomic DNA was precipitated with isopropanol, and washed with 75\% ethanol. Dried DNA pellets were suspended in $100 \mu \mathrm{l}$ of sterile double-distilled $\mathrm{H}_{2} \mathrm{O}$, and evaluated using a NanoDrop spectrophotometer (Nyxor Biotech, Paris, France; Caporaso et al. 2012). Subsequently, the V3-V4 regions of the 16S rRNA genes were amplified using the following procedures: initial denaturation at $95{ }^{\circ} \mathrm{C}$ for $2 \mathrm{~min}$, followed by 25 cycles of amplification at $95^{\circ} \mathrm{C}$ for $30 \mathrm{~s}, 55^{\circ} \mathrm{C}$ for $30 \mathrm{~s}$, and $72{ }^{\circ} \mathrm{C}$ for $30 \mathrm{~s}$, and final extension at $72{ }^{\circ} \mathrm{C}$ for $5 \mathrm{~min}$. The PCR primers employed were 341F: $5^{\prime}-$ CCTAYGGGRBGCASCAG-3' and 806R: 5'-GGAC TACNNGGGTATCTAAT-3'. The reaction mixture used was a $20 \mu \mathrm{l}$ mixture containing $4 \mu \mathrm{l}$ of $5 \times$ FastPfu Buffer, $2 \mu \mathrm{l}$ of $2.5 \mathrm{mM}$ dNTPs, $0.8 \mu \mathrm{l}$ of each primer $(5 \mu \mathrm{m}), 0.4 \mu \mathrm{l}$ of FastPfu Polymerase, and $10 \mathrm{ng}$ of template DNA, in triplicate. The PCR products were purified from agarose gels using a DNA gel extraction kit (TIANGEN Biotech, Beijing, China) according to the manufacturer's instructions, and their concentrations were determined using the QuantiFluor ${ }^{\mathrm{TM}}$ dsDNA system (Promega, Madison, WI, USA). Next, the purified amplicons were used to construct paired-end sequencing libraries, and then for paired-end 250-bp sequencing 
implementing the Illumina Hiseq 2500 platform (Campbell et al. 2010; Mao et al. 2012).

\section{Analyses of tags and operational taxonomic units (OTUs)}

First, the primer and adapter sequences were eliminated from the raw FASTQ files. Next, filtering was performed by eliminating sequences with more than $10 \%$ unknown nucleotides $(\mathrm{N})$ or $20 \%$ low-quality $(Q$ score $<20)$ bases. Additionally, these sequences that were identified to be mitochondrial DNA fragments were removed. Then, tags were generated according to the alignment between paired-end reads with an overlap of more than 10-bp and mismatch of less than $2 \%$. Subsequently, redundant tags were removed to generate unique tag dataset (Schloss et al. 2009). Meanwhile, DNA distance matrices were clustered to calculate the OTUs with sequence convergence greater than $97.0 \%$ in their individual microbial communities. Community diversity at the inter- and intra-group level was assessed using a combination of biascorrected Chaol richness estimator, abundance-based coverage estimator (ACE), Shannon and npShannon diversity indices, Simpson's diversity index, and coverage percentage. Based on the OTU expression profile, the beta diversity of different samples at OTU level was calculated. All the aforementioned analyses were conducted using the program MOTHUR (Kemp and Aller 2004; Schloss et al. 2009). The Principal Component Analysis (PCA) was analyzed based on the expression profile of OTUs at the taxonomic level using the R package (Team 2011).

\section{Taxonomic abundance profiling}

To annotate taxonomic information on the gut microbiota, we first used a Naive Bayes classifier to categorize the tags into different taxa against the Greengenes database (version 20101006) with a confidence threshold of 0.5 (DeSantis et al. 2006; Wang et al. 2007). Next, we classified the OTUs based on the tag annotation, in compliance with the mode principle, which requires more than $66 \%$ tags to rationalize the same level in the following order: domain, phylum, class, order, family, genus, and species. The abundances of each sample at different taxonomic levels were statistically calculated. All samples of two pig breed groups were used for chisquare test to investigate whether there was a significant difference between these two groups with respect to structure composition of microbial communities at the genus level via evaluating their eccentricity.

For the identification of differentially abundant taxa in multiple segments within the common pig group, we applied the linear discriminant analysis (LDA) effect size (LEFse) method (Segata et al. 2011). In brief, the Kruskal-Wallis rank sum test was performed to detect features with significantly different abundance among all groups. Next, the Wilcoxon rank sum test was conducted to detect features with significantly different abundance between two groups, and then LDA was performed to estimate the effect size of each feature. Additionally, a taxonomic cladogram representing the structure of the microbial community in each sample and their predominant bacteria was constructed to demonstrate the greatest differences in taxa among groups. For the identification of differentially abundant microbial taxa in the same segment among samples of different pig groups, Metastats was used (White et al. 2009).

\section{Molecular function prediction and pathway enrichment analysis}

Phylogenetic Investigation of Communities by Reconstruction of Unobserved States (PICRUSt; Langille et al. 2013) was applied to predict the functional enrichment of the microbial communities against the Kyoto Encyclopedia of Genes and Genomes (KEGG) database (Du et al. 2014). Correlation coefficients of the pathway enrichment for the samples of the common group were calculated using the Spearman method. The $\mathrm{R}$ package, edgeR (Robinson et al. 2010), was used to determine differentiating molecular functions and pathways under the control of $\log 2$ fold change $>2$ and FDR $<0.01$. Volcano plots and heatmaps were generated for the differentiating pathways.

Data availability The sequencing data from this study were deposited in the NCBI GEO database with the accession number SRP115844.

\section{Results}

\section{Sample tags and OTU analyses}

The tags for all samples added up to a count of 1,049,386, covering $479,469,744$ base pairs (Table 1). The average tag count per sample was 43,724 . The tag matrix merged convergently with a cut-off of $97 \%$ similarity to generate the OTU profile, resulting in an average of 84,601 OTUs in each sample (Table S1). Coverage was determined to be more than $90 \%$ in each sample. This ensured that the sequencing depth was sufficient for diversity evaluation, further confirmed by the saturated plateaus shown in the Shannon-Wiener curves (Fig. 1).

\section{Comparisons of microbiota among different samples}

Categorizing the Chinese Shanxi Black Pigs into the B group and Large White Pigs into the $\mathrm{W}$ group, we performed intraand inter-group comparisons. First, the different indices were calculated as described above to analyze the alpha diversities 
Table 1 Tag number, length, and quality of the segmented samples in both the B150 and W150 groups

\begin{tabular}{|c|c|c|c|c|c|c|c|c|}
\hline Sample ID & $\begin{array}{l}\text { Total tag } \\
\text { number }\end{array}$ & Total tag length (bp) & $\begin{array}{l}\text { Unique } \\
\text { tag number }\end{array}$ & $\begin{array}{l}\text { Unique tag total } \\
\text { length (bp) }\end{array}$ & Max length (bp) & Min length (bp) & N50 (bp) & N90 (bp) \\
\hline B150-D1 & 88,623 & $40,433,931$ & 52,613 & $23,967,697$ & 476 & 306 & 461 & 441 \\
\hline B150-D2 & 91,837 & $42,716,802$ & 38,480 & $17,863,060$ & 479 & 306 & 466 & 465 \\
\hline B150-D3 & 88,399 & $40,552,848$ & 42,099 & $19,281,415$ & 470 & 302 & 466 & 441 \\
\hline B150-I1 & 91,447 & $41,713,393$ & 41,167 & $18,739,771$ & 472 & 301 & 466 & 441 \\
\hline B150-I2 & 80,682 & $36,564,420$ & 30,913 & $13,984,205$ & 469 & 331 & 465 & 440 \\
\hline B150-I3 & 82,202 & $37,805,602$ & 34,706 & $15,883,041$ & 479 & 301 & 466 & 441 \\
\hline B150-J1 & 81,059 & $37,359,170$ & 38,857 & $17,863,867$ & 477 & 302 & 466 & 441 \\
\hline B150-J2 & 84,508 & $39,220,372$ & 35,081 & $16,253,351$ & 478 & 313 & 466 & 465 \\
\hline B150-J3 & 80,744 & $37,506,291$ & 32,553 & $15,093,518$ & 468 & 307 & 466 & 465 \\
\hline B150-C1 & 84,096 & $38,160,284$ & 53,209 & $24,115,126$ & 479 & 301 & 460 & 441 \\
\hline $\mathrm{B} 150-\mathrm{C} 2$ & 88,331 & $40,187,913$ & 61,757 & $28,080,561$ & 469 & 307 & 460 & 441 \\
\hline B150-C3 & 81,876 & $37,307,585$ & 53,237 & $24,219,617$ & 477 & 301 & 460 & 441 \\
\hline W150-D1 & 81,224 & $37,693,134$ & 32,933 & $15,240,330$ & 472 & 307 & 466 & 458 \\
\hline W150-D2 & 87,821 & $40,730,499$ & 40,031 & $18,501,276$ & 478 & 303 & 466 & 443 \\
\hline W150-D3 & 87,550 & $40,586,188$ & 36,607 & $16,918,874$ & 478 & 313 & 466 & 443 \\
\hline W150-I1 & 88,570 & $40,391,195$ & 54,827 & $24,948,553$ & 479 & 302 & 461 & 441 \\
\hline W150-I2 & 82,942 & $37,564,426$ & 34,010 & $15,365,410$ & 469 & 307 & 443 & 440 \\
\hline W150-I3 & 84,221 & $38,136,991$ & 49,891 & $22,574,109$ & 475 & 302 & 460 & 441 \\
\hline W150-J1 & 58,922 & $27,293,897$ & 24,918 & $11,518,753$ & 469 & 314 & 466 & 445 \\
\hline W150-J2 & 84,591 & $39,337,137$ & 37,751 & $17,524,297$ & 469 & 302 & 466 & 465 \\
\hline W150-J3 & 90,368 & $41,472,486$ & 45,688 & $20,888,465$ & 474 & 302 & 466 & 441 \\
\hline W150-C1 & 91,727 & $41,649,112$ & 62,786 & $28,484,681$ & 475 & 307 & 460 & 441 \\
\hline W150-C2 & 80,435 & $36,492,701$ & 55,366 & $25,105,543$ & 478 & 302 & 460 & 441 \\
\hline W150-C3 & 88,255 & $39,863,834$ & 59,906 & $27,054,224$ & 479 & 301 & 445 & 441 \\
\hline
\end{tabular}

of the samples (Table 2). When alpha diversity indices were compared among intra-group segments, as depicted in Fig. 1b, the smaller intestinal segments (D, J, and I) exhibited significantly higher $(p<0.01)$ Simpson's index values and lower $(p<0.01)$ Chao1, ACE, and Shannon's index values than the corresponding cecal samples, indicating that the cecum had a relatively richer community diversity for each pig breed. However, the alpha diversities of the corresponding intergroup segments were not significantly different between the Chinese Shanxi Black Pigs and Large White Pigs.

PCA was subsequently performed based on the OTU profile. As shown in the Fig. $1 \mathrm{c}$, the $\mathrm{C}$ segment samples both in $\mathrm{B}$ and $\mathrm{W}$ groups were obviously clustered into a small area and distinctly separated from these smaller intestinal samples, although two I segment samples of the $\mathrm{W}$ group were inter-mixed. Samples of the other segments exhibited obscure boundaries due to the variations within each segment, but there are, to some extent, certain intergroup differences between $\mathrm{B}$ and $\mathrm{W}$ groups in light of these non-overlapping regions in the same segments of individual samples.

To investigate specific differentiating taxa, LEFse and Metastats analyses were performed for intra- and inter-group comparisons, respectively (Fig. 2). In the intra-group analysis, the $\mathrm{C}$ and D segments of the B group presented 24 and 17 specifically enriched taxa, respectively, whereas I and $\mathrm{J}$ segments had only 6 and 2 respectively, suggesting that there were more unique taxa in the $\mathrm{C}$ and $\mathrm{D}$ segments than that in other segments with respect to microbial composition (Fig. $2 \mathrm{a}-\mathrm{c}$ ). A similar pattern was observed in the $\mathrm{W}$ group (Fig. $2 \mathrm{~d}-\mathrm{f})$. More details for the differentiating taxa were summarized in Table S2.

Based on the inter-group comparisons, the $\mathrm{D}$ segment of the W group exhibited a higher prevalence of Methylobacteriaceae, whereas in the B group, the genera Mycoplasma, Micrococcus, and Collinsella were more enriched. Furthermore, the genus Streptococcaceae was more abundant in the I segment of the W group, while the genus Veillonella was more enriched in the corresponding segment of the B group. As for the J segment, the only significant difference between the groups was that the B group exhibited a higher abundance of Moraxella. Finally, in the $\mathrm{C}$ segment, the $\mathrm{W}$ group was enriched with the genera Buchnera, Mitsuokella, and Anaerovibrio, while the B group showed a higher prevalence of the genus Flexispira. The corresponding results about the Metastats analysis were listed in Tables S3-S6. 


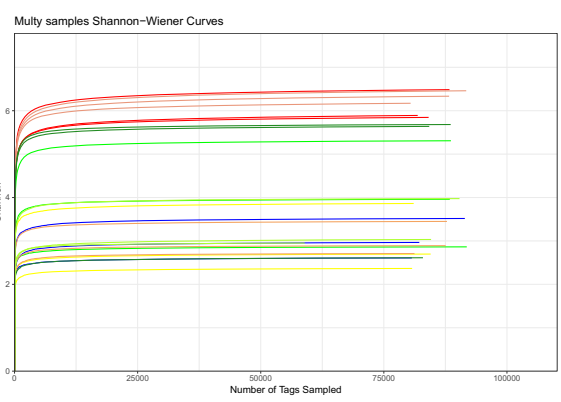

a
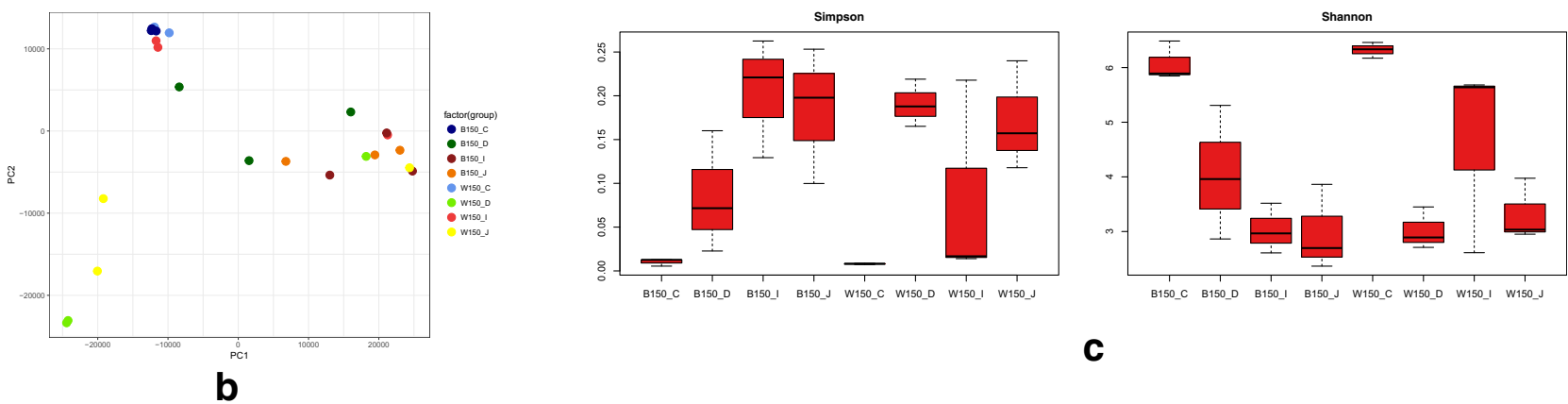

C

Fig. 1 Profile of the intestinal microbiota. a. Correlation coefficients of the segmented samples in both the B25 and B70 groups. b. Multi-sample Shannon-Wiener curves of segmented samples in both the B25 and B70 groups. Shannon, ACE, Simpson, and Chaol indices of the merged segments in both the B25 and B70 groups. (In both groups, the small intestinal segments exhibited significantly higher $(p<0.01)$ Simpson index values and lower $(p<0.01)$ Chao1, ACE, and Shannon index values than in their respective cecum). c. PCA plot of the segmented samples based on the OTU profiling
Table 2 Alpha diversities of the segmented samples in both the B150 and W150 groups

\begin{tabular}{|c|c|c|c|c|c|c|}
\hline Sample & Chaol & ACE & Shannon & npShannon & Simpson & Coverage \\
\hline B150-C1 & $22,860.4762$ & $48,236.2775$ & 5.846312 & 5.995333 & 0.012758 & 0.943743 \\
\hline B150-C2 & $34,666.361$ & $71,931.9556$ & 6.485726 & 6.648571 & 0.005468 & 0.922405 \\
\hline B150-C3 & $25,149.0037$ & $51,895.187$ & 5.89118 & 6.063425 & 0.012571 & 0.934938 \\
\hline B150-D1 & $11,667.2844$ & $21,653.6577$ & 5.307968 & 5.398138 & 0.022712 & 0.971599 \\
\hline B150-D2 & 5583.65517 & 8241.90147 & 2.863535 & 2.946685 & 0.160083 & 0.985583 \\
\hline B150_D3 & 7666.16404 & $13,201.4048$ & 3.959762 & 4.049414 & 0.07162 & 0.980328 \\
\hline B150-I1 & 9145.64066 & $13,877.4467$ & 3.517401 & 3.639182 & 0.129343 & 0.976259 \\
\hline B150-I2 & 6200.93031 & $10,139.0371$ & 2.60861 & 2.71888 & 0.220959 & 0.981272 \\
\hline B150-I3 & 7741.86416 & $13,042.0993$ & 2.965487 & 3.08883 & 0.262569 & 0.977823 \\
\hline B150-J1 & 8997.861 & $14,091.9633$ & 3.862994 & 3.997356 & 0.099813 & 0.972033 \\
\hline B150-J2 & 6433.43451 & $10,824.5524$ & 2.697345 & 2.807029 & 0.197825 & 0.98128 \\
\hline B150-J3 & 5228.33798 & 9049.26988 & 2.366332 & 2.469578 & 0.253232 & 0.983305 \\
\hline W150-C1 & $37,808.9312$ & $81,672.5059$ & 6.459794 & 6.640852 & 0.007221 & 0.91732 \\
\hline W150-C2 & $26,361.3135$ & $57,835.8843$ & 6.171876 & 6.306373 & 0.00814 & 0.939479 \\
\hline W150-C3 & $30,830.9647$ & $65,074.0218$ & 6.334703 & 6.499367 & 0.008716 & 0.927415 \\
\hline W150-D1 & 5128.84906 & 7727.31829 & 2.709457 & 2.792045 & 0.218989 & 0.985719 \\
\hline W150-D2 & 7014.77083 & $10,306.4432$ & 3.449513 & 3.547836 & 0.165138 & 0.981018 \\
\hline W150-D3 & 5394.02317 & 8610.65538 & 2.89118 & 2.975042 & 0.18784 & 0.985243 \\
\hline W150-I1 & $11,747.6126$ & $24,960.2685$ & 5.682058 & 5.75519 & 0.016717 & 0.972925 \\
\hline W150-I2 & 6073.84884 & 9767.38734 & 2.612484 & 2.727035 & 0.21784 & 0.980999 \\
\hline W150-I3 & $14,680.4572$ & $30,521.4439$ & 5.639194 & 5.728145 & 0.013989 & 0.966908 \\
\hline W150-J1 & 4945.87678 & 9453.02828 & 2.950896 & 3.054985 & 0.157178 & 0.980313 \\
\hline W150-J2 & 8837.85327 & $14,225.2402$ & 3.033529 & 3.17835 & 0.239971 & 0.974134 \\
\hline W150-J3 & $10,251.2287$ & $20,148.5248$ & 3.97502 & 4.08147 & 0.117862 & 0.97612 \\
\hline
\end{tabular}


a
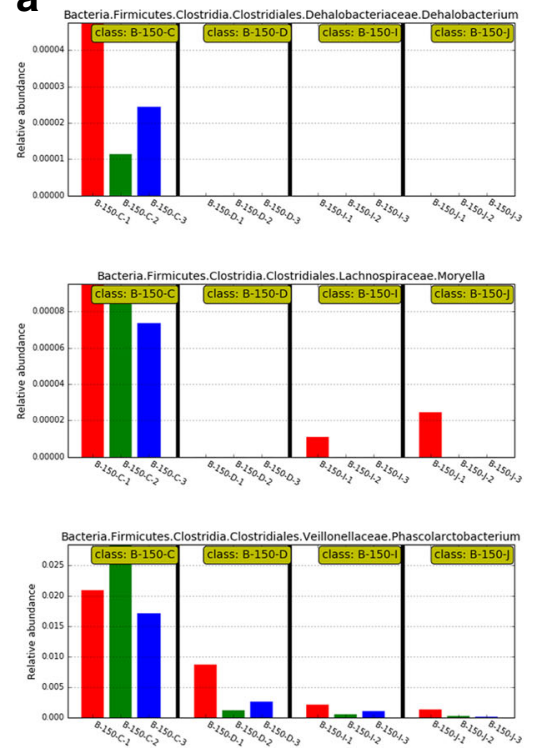

d
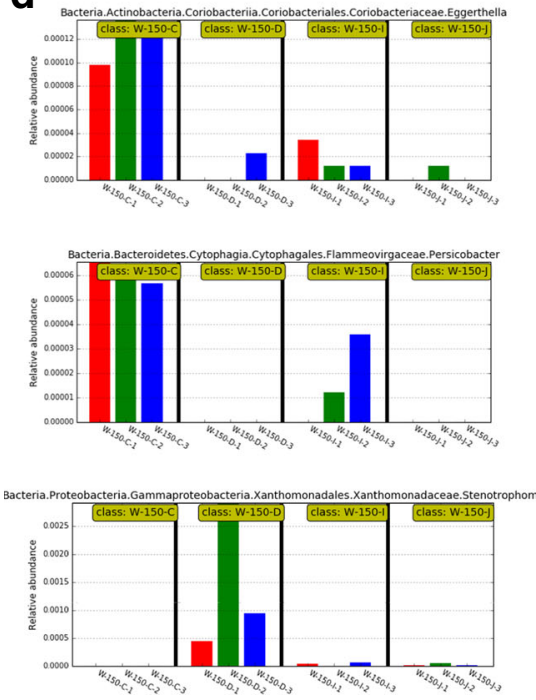

Fig. 2 LEFse analysis revealed differentiated taxon. a. Abundances of selected differentiated taxa in B150 group. b. LDA scores of differentiated taxa in B150 group. c. Cladogram of differentiated taxa of

\section{Microbiotic profiling of distinct intestinal segments}

The OTU profile was used to determine taxon abundance in the samples. At the phylum level, the predominant phyla in all samples were Firmicutes, Proteobacteria, and Bacteroidetes (Fig. 3a and Table S7), and the average proportions of which were $44.0 \%, 33.9 \%$, and $18.3 \%$, respectively. Further inspection presented that the abundances of phylum Firmicutes in the D2 (4.7\%) and J3 (12.5\%) samples of the B group were surprisingly low, while the same phylum in the D1 and J3 samples of were identified to account for more than $80 \%$ compared to the total samples in the $\mathrm{W}$ group. In spite of these issues, Firmicutes was considered as the most prevalent phylum in all samples in light of their relatively higher average b

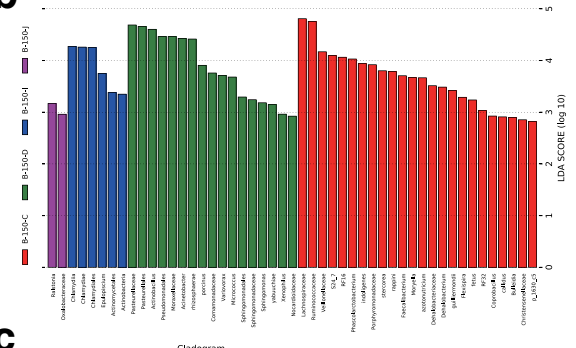

C
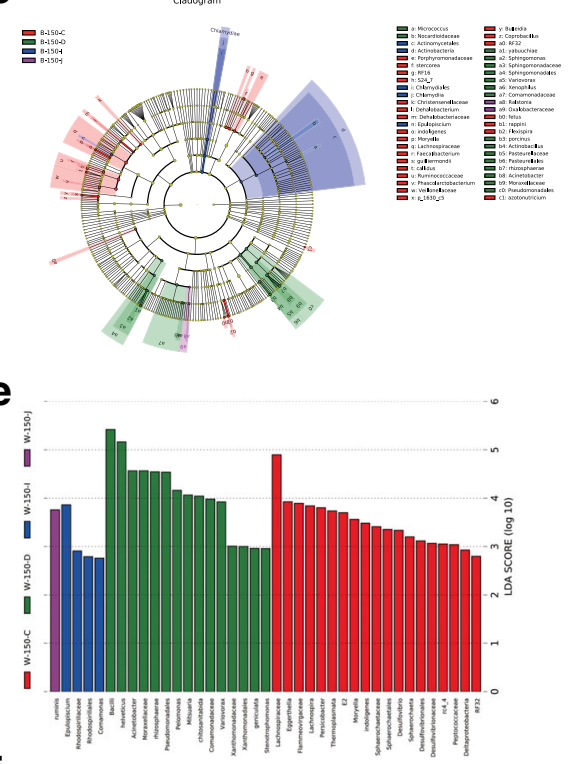

f

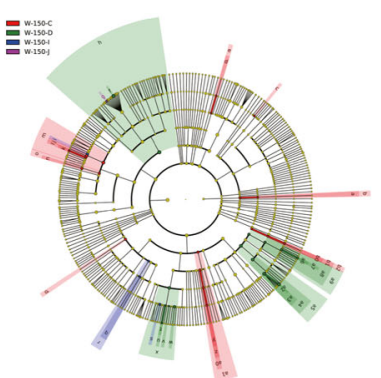

the segments in in B150 group. d. Abundances of selected differentiated taxa in W150 group. e. LDA scores of differentiated taxa in W150 group. f. Cladogram of differentiated taxa of the segments in in W150 group

abundances (Fig. 3a). Charmingly, the distributions of Proteobacteria and Bacteroidetes, to some extent, showed a mutually exclusive pattern. For instance, Proteobacteria (43.7\%) was dominant in the relatively smaller intestinal segments, whereas Bacteroidetes (46.1\%) was dominant in the cecal samples. Owing to the unexpectedly low abundances of Firmicutes in the D2 and J3 samples, the prevalence of Proteobacteria ( $93.0 \%$ and $86.4 \%$, respectively) in these samples was the highest across all samples.

Among the genera (Fig. 3b and Table S8), Lactobacillus was predominant, constituting an average of $33.2 \%$ in all samples. This result suggested that genus Lactobacillus had thrived in the guts of both breeds. In contrast to other intestinal segment samples, the generic composition in the cecal 

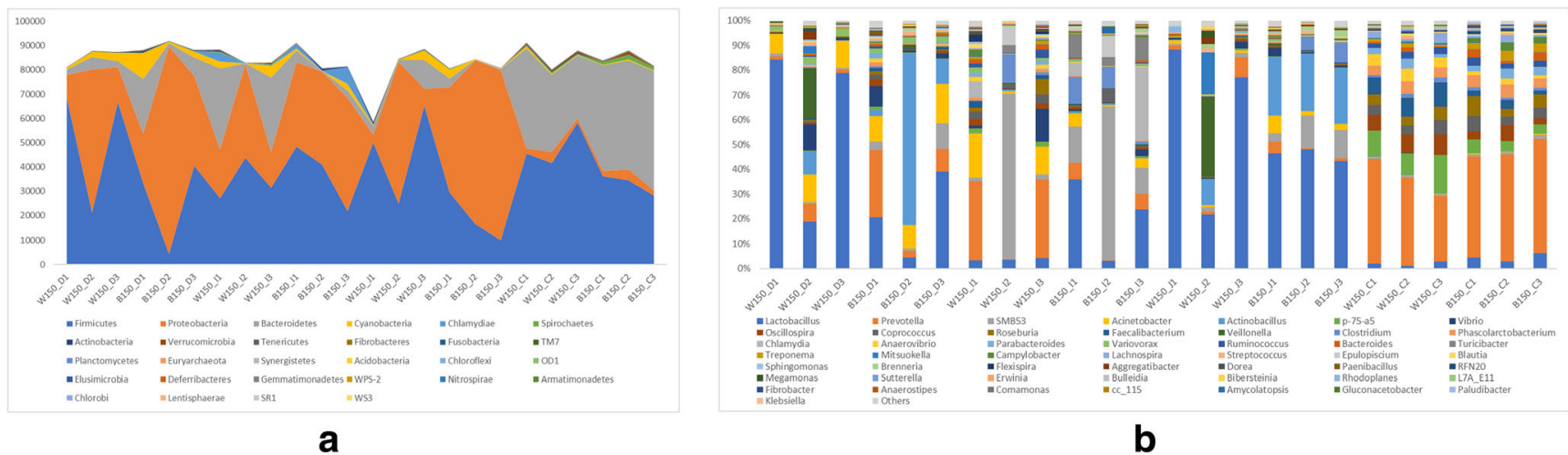

Fig. 3 Relative abundances of sequences belonging to different phyla and genera. a. Phylum distribution of the segmented samples in both the B150 and W150 groups. b. Genera distribution of the segmented samples in both the B150 and W150 groups

samples of the two breeds appeared to be more consistent. Prevotella was the key genus in the cecal samples, and it was not prevalent in the smaller intestinal samples (excluding I1 and I3) of the W group. Similar to analysis regarding the alpha diversities, chi-square test showed that no significant difference between these two groups at the genus level was observed (Table S9).

\section{Molecular function prediction and pathway enrichment analyses}

Using the present OTU data, PICRUSt was applied to determine the potential pathway enrichment of intestinal samples via annotation against the KEGG database. In all samples, the majority of OTUs were assigned to 24 gene families, which were mainly involved in signal transduction, membrane transport, nutrient metabolism, energy metabolism, and genetic material processing (Table S10). The prevalence of pathways at the KEGG A class level was similar for different samples (Fig. 4a). Notably, metabolism, environmental information processing, and genetic information processing were the most enriched functional modules. At the B class level, comparisons of the predicted KEGG functions among all samples revealed that extremely few OTUs in the cecal samples were involved in energy metabolism, biosynthesis of secondary metabolites, and xenobiotics biodegradation and metabolism
(Fig. 4b). The D segment in $\mathrm{W}$ group and $\mathrm{J}$ segment in $\mathrm{B}$ group had fewer OTUs potentially related to immune system compared to their counterparts in the other groups. Furthermore, relatively fewer OTUs associated with xenobiotics biodegradation and metabolism were predicted to be present in the $\mathrm{J}$ segment of $\mathrm{W}$ group.

\section{Discussion}

In recent years, emerging studies have focused on intestinal microbiota of livestock. Studies on microbiota of gut segments of the horse, pig, camel, cow, etc. have been conducted previously. Intestinal microbes are considered to be dynamic. Their dynamic distributions were indicated to be influenced by the host, beginning at birth, and reaching stability at maturity (Lee et al. 2017; Rosen and Palm 2017; Shen 2017; Yamashiro 2017; Lopetuso et al. 2018). In this study, two different mature pig breeds, i.e., Chinese Shanxi Black Pigs and Large White Pigs, were selected as the objects, and samplings of their intestinal contents rather than fecal samples were performed to investigate the potential structure and predicted functions of microbial communities in distinct intestinal segments.

In our study, the analysis of taxa in both the Chinese Shanxi Black Pigs and Large White Pigs revealed the dominant phyla to be Firmicutes and Bacteroidetes, whose prevalence has been a

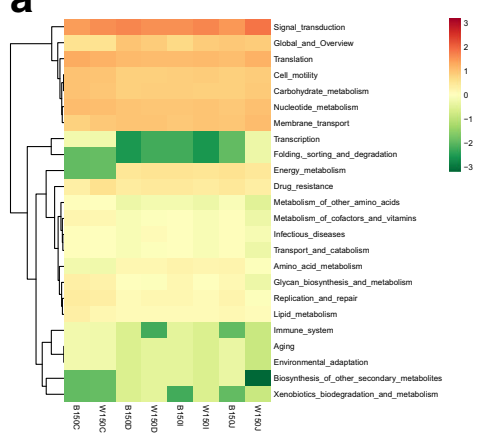

b

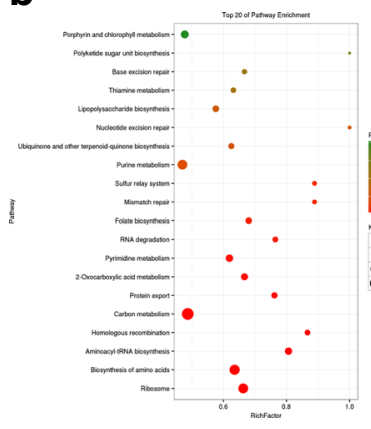

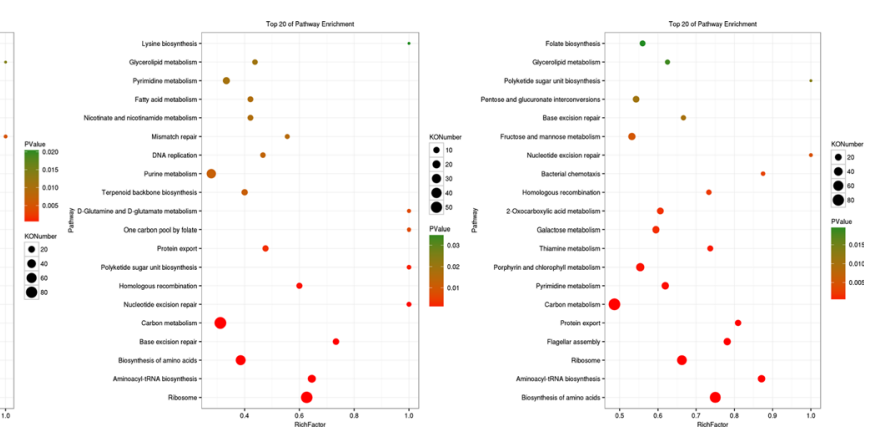

Fig. 4 Inter-group differentiated molecular functions and pathways. a. Pathway count heatmap of the distinctive segments in the B150 and W150 groups. b. Differentiated pathways based on metastat-revealed differentiated of the J, I, and C segments between the B150 and W150 groups 
shown to fluctuate in previous studies on mammalian intestinal microbiota. Nevertheless, the microbial communities examined in this study exhibited certain distinct differences, compared with those in previous porcine studies, which might have resulted from feeding and husbandry conditions. For instance, the distribution of Firmicutes observed in our study was not completely consistent with that in previous studies. Generally, Firmicutes is the most prevalent gut microbial phylum in most mammals, and constitutes the largest proportion of the gut microbiome. This phylum has been generally recognized to be associated with fat metabolism and energy resorption, thereby suggesting that Firmicutes was probably involved in the development of diabetes and obesity (Schoster et al. 2013; Kallus and Brandt 2012; Machado and Cortez-Pinto 2012; Bressa et al. 2017; Most et al. 2017). An earlier study reported that the porcine large intestine, especially the cecum, has a higher proportion of Firmicutes than the small intestine, suggesting that the cecum, rather than the small intestine, might contribute substantially to fat deposition (Mao et al. 2015). In contrast to these previous studies, interestingly, our study presented that the differences in the prevalence of Firmicutes between the cecum and small intestine were not as profound as to infer such functions. Composition analysis showed that the cecum had larger proportions of Bacteroidetes, and the small intestine had larger proportions of Proteobacteria. Certain previous studies have reported the relatedness of Proteobacteria with inflammation. Additionally, this phylum was reported to harbor various metabolic functions, which might explain the fact that the microbial function prediction in this study did not mean many enriched metabolic pathways in the cecum, in contradiction to certain other studies, although we observed greater species diversities in the ceca of both the black and white porcine breeds as well.

Notably, Lactobacillus, the member of most common probiotics, was observed to be the predominant genus in both adult porcine breeds. The genus Lactobacillus was previously reported to exhibit a mutualistic relationship with the gut, by forming biofilms to facilitate maintenance during harsh conditions, as well as to provide barriers to the host against potential infections (Ahn et al. 2018). In one of our previous studies, we reported that pigs at the nursery stage do not contain Lactobacillus. This result suggested that administration of prebiotics required several months to exert effects, and pathogen invasion and nutritional problems for pigs at the nursery stage should be addressed more cautiously, as their gut microbiota is still vulnerable. Accordingly, the development of new prebiotics or novel methods might facilitate the early colonization of mutualistic microbes in porcine guts at vulnerable developmental stages, such as piglets with immature immune systems, and further promote the production of the pig farming industry.

Further analyses revealed that several differentiating taxa were identified between the two porcine breeds. In this study, the duodenum and cecum contained more unique taxa than the jejunum and ileum. For the white pig breed, the cecum was characterized by the presence of the genera Eggerthella, Persicobacter, and Sphaerochaeta, and the duodenum was specifically enriched with the genera Mitsuaria and Stenotrophomonas. However, the ceca of the black pigs were characterized by the presence of the genera Dehalobacterium, Moryella, and Phascolarctobacterium, and the duodena, by the genera Mitsuaria and Stenotrophomonas. Contrastingly, its jejunum and ileum hardly contained distinctive genera. Accordingly, the differences of structure composition of microbial communities might drive the possible distinctive host features. However, it is necessary to further investigate the functional roles of these unique microbial species in respective econiches and the potential contributions to phenotypic differences of hosts.

In conclusion, we examined the microbial composition in four distinct intestinal segments of Chinese Shanxi Black Pigs and Large White Pigs, and successfully identified their differentially distributed genera as biomarker candidates for further studies. Intra- and inter-groups comparisons shed light on the differences of structure compositions and predicted functions of microbial communities in distinct intestinal segments between two pig breeds, and provided some insight into the potential relation between intestinal microbiota and host features. In the further studies, it was of interest to perform other omics-based trials, such as genomics and metagenomics, to explore the individual potentials in the microbial communities, or the ecological roles of both dominant and rare prokaryotes, respectively.

Acknowledgments We would like to thank Editage (available at www. editage.cn) for English language editing. We would like to thank Guangzhou Gene denovo Biotechnology Co., Ltd. for 16S rRNA gene sequences and data analyses. The authors wish to thank the anonymous reviewers for their valuable comments and suggestions, which were helpful in improving our manuscript.

Funding information The project was funded by the Foundation of Science and Technology Innovation Team of Shanxi Province (201705D131028-19), the Fund for Shanxi 1331 Project, supported by the Program for Sanjin Scholar $(2016,2017)$ and Postdoctoral fund of Shanxi Agricultural University.

\section{Compliance with ethical standards}

Conflict of interest The authors declare that they have no conflict of interest.

Ethical approval Drug Safety Evaluation Centre of China Institute for Radiation Protection provided necessary experimental facilities and animal use certificate (SYXK 2013-0002) to support this experimentation on animals. All applicable international, national, and/or institutional guidelines for the care and use of animals were followed. 
Open Access This article is distributed under the terms of the Creative Commons Attribution 4.0 International License (http:// creativecommons.org/licenses/by/4.0/), which permits unrestricted use, distribution, and reproduction in any medium, provided you give appropriate credit to the original author(s) and the source, provide a link to the Creative Commons license, and indicate if changes were made.

Publisher's note Springer Nature remains neutral with regard to jurisdictional claims in published maps and institutional affiliations.

\section{References}

Ahn KB, Baik JE, Park OJ, Yun CH, Han SH (2018) Lactobacillus plantarum lipoteichoic acid inhibits biofilm formation of Streptococcus mutans. PLoS One 13:e0192694

Barko PC, McMichael MA, Swanson KS, Williams DA (2017) The gastrointestinal microbiome: a review. J Vet Intern Med

Bressa C, Bailén-Andrino M, Pérez-Santiago J, González-Soltero R, Pérez M, Montalvo-Lominchar MG, Maté-Muñoz JL, Domínguez R, Moreno D, Larrosa M (2017) Differences in gut microbiota profile between women with active lifestyle and sedentary women. PLoS One 12:e171352

Campbell BJ, Polson SW, Hanson TE, Mack MC, Schuur EA (2010) The effect of nutrient deposition on bacterial communities in Arctic tundra soil. Environ Microbiol 12:1842-1854

Caporaso JG, Lauber CL, Walters WA, Berg-Lyons D, Huntley J, Fierer N, Owens SM, Betley J, Fraser L, Bauer M, Gormley N, Gilbert JA, Smith G, Knight R (2012) Ultra-high-throughput microbial community analysis on the Illumina HiSeq and MiSeq platforms. ISME J 6: $1621-1624$

D'Argenio V, Salvatore F (2015) The role of the gut microbiome in the healthy adult status Clinica chimica acta. Clin Chim Acta 451:97102

DeSantis TZ, Hugenholtz P, Larsen N, Rojas M, Brodie EL, Keller K, Huber T, Dalevi D, Hu P, Andersen GL (2006) Greengenes, a chimera-checked 16S rRNA gene database and workbench compatible with ARB. Appl Environ Microbiol 72:5069-5072

Du J, Yuan Z, Ma Z, Song J, Xie X, Chen Y (2014) KEGG-PATH: Kyoto encyclopedia of genes and genomes-based pathway analysis using a path analysis model. Mol BioSyst 10:2441-2447

Ellegaard KM, Engel P (2016) Beyond 16S rRNA community profiling: intra-species diversity in the gut microbiota. Front Microbiol 7:1475

Fang S, Xiong X, Su Y, Huang L, Chen C (2017) 16S rRNA gene-based association study identified microbial taxa associated with pork intramuscular fat content in feces and cecum lumen. BMC Microbiol $17: 162$

George S, Oh Y, Lindblom S, Vilain S, Rosa AJM, Francis DH, Brözel VS, Kaushik RS (2007) Lectin binding profile of the small intestine of five-week-old pigs in response to the use of chlortetracycline as a growth promotant and under gnotobiotic conditions. J Anim Sci 85: $1640-1650$

He D, Zou T, Gai X, Ma J, Li M, Huang Z, Chen D (2017) MicroRNA expression profiles differ between primary myofiber of lean and obese pig breeds. PLoS One 12:e181897

Hillman ET, Lu H, Yao T, Nakatsu CH (2017) Microbial ecology along the gastrointestinal tract. Microbes Environ 32:300-313

Inatomi T, Amatatsu M, Romero-Perez GA, Inoue R, Tsukahara T (2017) Dietary probiotic compound improves reproductive performance of porcine epidemic diarrhea virus-infected sows reared in a japanese commercial swine farm under vaccine control condition. Front Immunol 8:1877

Kallus SJ, Brandt LJ (2012) The intestinal microbiota and obesity. J Clin Gastroenterol 46:16-24
Kemp PF, Aller JY (2004) Bacterial diversity in aquatic and other environments: what $16 \mathrm{~S}$ rDNA libraries can tell us. FEMS Microbiol Ecol 47:161-177

Kim J, Kim J, Kim Y, Oh S, Song M, Choe JH, Whang K, Kim KH, Oh S (2018) Influences of quorum-quenching probiotic bacteria on the gut microbial community and immune function in weaning pigs. Anim Sci J 89:412-422

Kundu P, Blacher E, Elinav E, Pettersson S (2017) Our gut microbiome: the evolving inner self. Cell 171:1481-1493

Langille MG, Zaneveld J, Caporaso JG, McDonald D, Knights D, Reyes JA, Clemente JC, Burkepile DE, Vega Thurber RL, Knight R, Beiko RG, Huttenhower C (2013) Predictive functional profiling of microbial communities using 16S rRNA marker gene sequences. Nat Biotechnol 31:814-821

Lee YY, Hassan SA, Ismail IH, Chong SY, Raja Ali RA, Amin Nordin S, Lee WS, Majid NA (2017) Gut microbiota in early life and its influence on health and disease: a position paper by the malaysian working group on gastrointestinal health. J Paediatr Child Health 53: $1152-1158$

Lee SH, Hur MH, Lee EA, Hong KC, Kim JM (2018) Genomic characterization of the porcine CRTC3 and the effects of a nonsynonymous mutation p.V515F on lean meat production and belly fat. Meat Sci 137:211-215

Ley RE, Hamady M, Lozupone C, Turnbaugh PJ, Ramey RR, Bircher JS, Schlegel ML, Tucker TA, Schrenzel MD, Knight R, Gordon JI (2008) Evolution of mammals and their gut microbes. Science 320:1095-9203

Lopetuso LR, Petito V, Graziani C, Schiavoni E, Paroni Sterbini F, Poscia A, Gaetani E, Franceschi F, Cammarota G, Sanguinetti M, Masucci L, Scaldaferri F, Gasbarrini A (2018) Gut microbiota in health, diverticular disease, irritable bowel syndrome, and inflammatory bowel diseases: time for microbial marker of gastrointestinal disorders. Dig Dis 36:56-65

Machado MV, Cortez-Pinto H (2012) Gut microbiota and nonalcoholic fatty liver disease. Ann Hepatol 11:440-449

Mao S, Zhang R, Wang D, Zhu W (2012) The diversity of the fecal bacterial community and its relationship with the concentration of volatile fatty acids in the feces during subacute rumen acidosis in dairy cows. BMC Vet Res 8:237

Mao S, Zhang M, Liu J, Zhu W (2015) Characterising the bacterial microbiota across the gastrointestinal tracts of dairy cattle: membership and potential function. Sci Rep 5:16116

Most J, Goossens GH, Reijnders D, Canfora EE, Penders J, Blaak EE (2017) Gut microbiota composition strongly correlates to peripheral insulin sensitivity in obese men but not in women. Benefic Microbes 8:557-562

Robinson MD, McCarthy DJ, Smyth GK (2010) edgeR: a Bioconductor package for differential expression analysis of digital gene expression data. Bioinformatics 26:139-140

Rosen CE, Palm NW (2017) Functional classification of the gut microbiota: the key to cracking the microbiota composition code: functional classifications of the gut microbiota reveal previously hidden contributions of indigenous gut bacteria to human health and disease. BioEssays 39

Schloss PD, Westcott SL, Ryabin T, Hall JR, Hartmann M, Hollister EB, Lesniewski RA, Oakley BB, Parks DH, Robinson CJ, Sahl JW, Stres B, Thallinger GG, Van Horn DJ, Weber CF (2009) Introducing mothur: open-source, platform-independent, community-supported software for describing and comparing microbial communities. Appl Environ Microbiol 75:7537-7541

Schoster A, Arroyo LG, Staempfli HR, Weese JS (2013) Comparison of microbial populations in the small intestine, large intestine and feces of healthy horses using terminal restriction fragment length polymorphism. BMC Res Notes 6:91 
Segata N, Izard J, Waldron L, Gevers D, Miropolsky L, Garrett WS, Huttenhower C (2011) Metagenomic biomarker discovery and explanation. Genome Biol 12:R60

Shen TD (2017) Diet and gut microbiota in health and disease. Nestle Nutr Inst Workshop Ser 88:117-126

Sommer F, Backhed F (2013) The gut microbiota-masters of host development and physiology. Nat Rev Microbiol 11:227

Team RDC (2011) R: A language and environment for statistical computing. The R Foundation for Statistical Computing, Vienna

Tian Y, Nichols RG, Cai J, Patterson AD, Cantorna MT (2017) Vitamin A deficiency in mice alters host and gut microbial metabolism leading to altered energy homeostasis. J Nutr Biochem 54:28-34

Wang Q, Garrity GM, Tiedje JM, Cole JR (2007) Naive Bayesian classifier for rapid assignment of rRNA sequences into the new bacterial taxonomy. Appl Environ Microbiol 73:5261-5267
White JR, Nagarajan N, Pop M (2009) Statistical methods for detecting differentially abundant features in clinical metagenomic samples. PLoS Comput Biol 5:e1000352

Woo PC, Lau SK, Teng JL, Tse H, Yuen KY (2008) Then and now: use of $16 \mathrm{~S}$ rDNA gene sequencing for bacterial identification and discovery of novel bacteria in clinical microbiology laboratories. Clin Microbiol Infect 14:908-934

Yamashiro Y (2017) Gut microbiota in health and disease. Ann Nutr Metab 71:242-246

Zou C, Li S, Deng L, Guan Y, Chen D, Yuan X, Xia T, He X, Shan Y, Li C (2017) Transcriptome analysis reveals long intergenic noncoding rnas contributed to growth and meat quality differences between Yorkshire and Wannanhua pig. Genes (Basel) 18:8 